\title{
Impact of Security Cameras on Electromagnetic Environment in Far and Near-Field
}

\author{
Stanislav Kovář, Jan Valouch, Hana Urbančoková and Milan Adámek \\ Faculty of Applied Informatics \\ Tomas Bata University in Zlín \\ Zlín, Czech republic \\ skovar@fai.utb.cz,valouch@fai.utb.cz, urbancokova@fai.utb.cz, adamek@fai.utb.cz
}

\begin{abstract}
Electromagnetic interference constitutes a serious problem for the design and operation of electronic and electrical security devices or systems because the impairment of the device function gives rise security complications. The complications may be associated with false alarms, errors in control of connected devices, image defects, losses of communication. The main purpose of the experiment reported here was to analyze and compare the level of the electromagnetic interference in a near and far field. In addition, the article also provides an interesting comparison of the results obtained by measuring in the Gigahertz Transverse Electromagnetic cell and semi-anechoic chamber.
\end{abstract}

Keywords-Electromagnetic interference; semi-anechoic chamber; gigahertz transverse electromagnetic cell; closed circuit television; near-field; far-field

\section{INTRODUCTION (HEADING 1)}

Electromagnetic interference (EMI) remains a serious problem of security cameras because this harmful disturbance affects both the device itself and all electronic devices located in the vicinity. Electromagnetic interference is a scientific discipline that is defined as the process of transmitting the generated signal through electromagnetic coupling into a disturbed device or system. [1] To achieve adequate levels of electromagnetic interference is necessary to pay attention to all components during development of the facility.

The paper refers to the findings mentioned in [3], which are dedicated to the Closed Circuit Television (CCTV) from the perspective of electromagnetic interference. However, the publication is aimed at the entire system, not just security cameras. Previous research based on level measurement of interference in the far field, thus providing an opportunity to expand the issue.

Because little attention is paid to the security cameras, the authors expanded the information specified in [3] and determine the effects of electromagnetic interference on the near and far field. The main purpose of this paper is to describe and analyze the impact of the electromagnetic interference on the selected device.

According to [8], to determine the areas of the requirements for electromagnetic compatibility (EMC) of camera systems is necessary to use a series of CCTV industry standards: CSN EN 50132 and the corresponding basic, tribal and product standards. Technical standard CSN EN 50132-1 specifies only briefly requirements on compliance of conditions established in following standards:

- $\quad$ CSN EN 50130-4 ed. 2 Alarm systems - Part 4: Electromagnetic compatibility - Product family standard: Immunity requirements for components of fire, intruder, hold up, CCTV, access control and social alarm systems.

- CSN EN 61000-6-3 Electromagnetic compatibility (EMC) - Part 6-3: Generic standards - Emission standard for residential, commercial and lightindustrial environments.

Currently, measurement of electromagnetic emission is performed in accordance with product standard EN 55022 ed. 3.

\section{EQUIPMENT UNDER TEST}

Closed Circuit Television is a surveillance and security system designed to capture, compile, transmit and store video information. Image information captured by the camera is processed and sent via a transmission medium to a control or display unit. For the purpose of the paper, CCTV contains the IP camera and a display/control device (laptop). Nevertheless, the test was carried out on the camera and the display device is used only to verify the activity of the camera. Vivotek FD8136B F3 was selected to test as a representative of the security cameras.

\section{MEASURING EQUIPMENT}

Measuring device means a device designed to obtain information about the status of electromagnetic interference or susceptibility selected device. Testing IP cameras were performed in the laboratories of Tomas Bata University in Zlin. Since the different types of assays were the aim, measurements were executed under various conditions and measurement techniques.

\section{A. Semi-anechoic chamber}

Semi-anechoic chamber for its shielding properties offers an almost perfect environment for testing the devices or systems in terms of electromagnetic compatibility. The semi- 
anechoic chamber allows to realize measurement in a wide frequency range without the influence of external electromagnetic fields, which would degrade the measurement results. Walls of the chambers are filled by pyramidal absorbers that eliminate internal reflections.

This equipment was used to measure the disturbance of camera:

- ESU 8 (Rohde \& Schwarz) - Test receiver operating range from $20 \mathrm{~Hz}$ to $8 \mathrm{GHz}$,

- CBL 6112 - log-periodic antenna (Figure 1),

- OSP 130 (Rohde \& Schwarz) - switching and control unit,

- OSP 150 (Rohde \& Schwarz) - switching and control unit,

- $\quad$ EMC32 (Rohde \& Schwarz) - EMC measurement software.

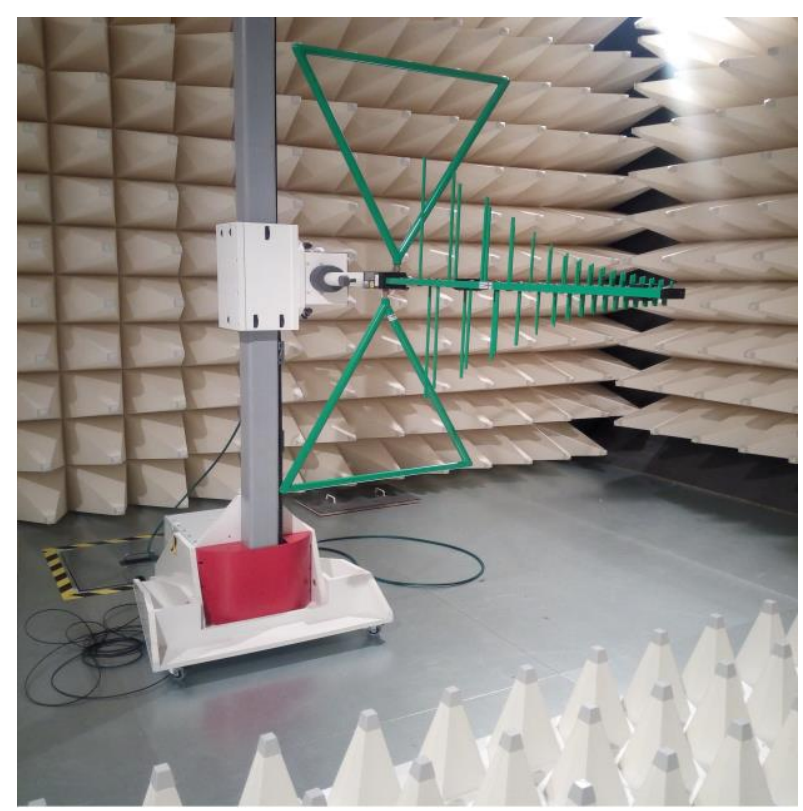

Figure 1. Bilogarithmic-periodic antenna. [3]

The bilogarithmic-periodic antenna was located inside the chamber and other measuring equipment outside the chamber to avoid distortion of the results. The security camera was powered via Power over Ethernet (PoE) adapter connected to the power grid. To determine the effect of the adapter on the overall level of interference, it was performed a few tests without PoE. Frequency range allows to measure from $30 \mathrm{MHz}$ to $1 \mathrm{GHz}$ thereby meets the requirements of the standard. IP camera was connected to the laptop via unshielded twisted pair (UTP), thus influencing the results by external parasitic signals.

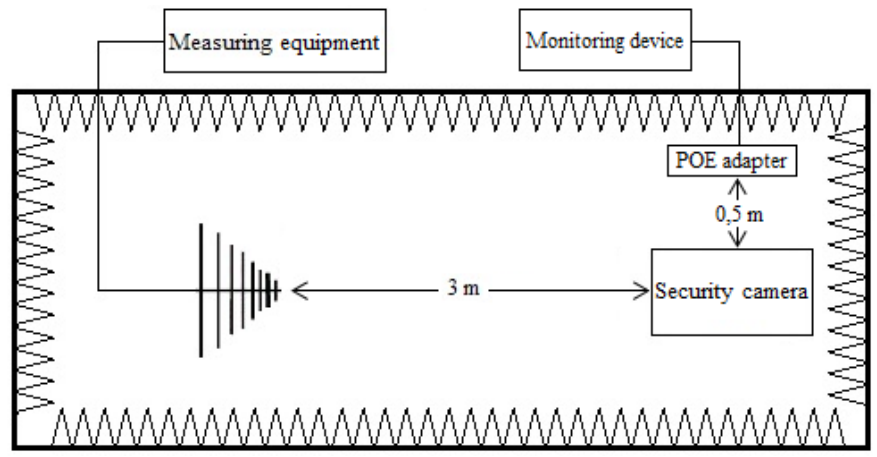

Figure 2. Disposition of equipment under test in semi-anechoic chamber.

\section{B. GTEM cell}

GTEM cell represents another test chamber to determine the level of electromagnetic compatibility. GTEM cell removes a limiting factor of TEM cells and allows to measure the frequencies above $1 \mathrm{GHz}$ which is also caused by the shape of the chamber. The broadband impedance matching at higher frequencies is ensured by the absorption pyramids while zero reflections in the lower frequency provide an internal resistor network.

For the purpose of the measurement, the following equipment was needed:

- EMC-GTEM-250 (Frankonia),

- $\quad$ ESPI Test receiver (Rohde \& Schwarz) - operating frequency of $9 \mathrm{kHz}$ to $7 \mathrm{GHz}$,

- EFS-10 (Frankonia) - measuring probe.

GTEM cell was connected to a test receiver used to display the course of electromagnetic interference in individual frequencies. As in the case of a semi-anechoic chamber, the frequency range was set from $30 \mathrm{MHz}$ to $1 \mathrm{GHz}$ in order to compare measurements. To decide on the intensity of the electromagnetic field, the probe must be placed with the camera in the chamber. As can be seen in the Figure 3, the probe was connected to the laptop that display the values of the electromagnetic field. However, these values are not relevant for the purpose of the paper.

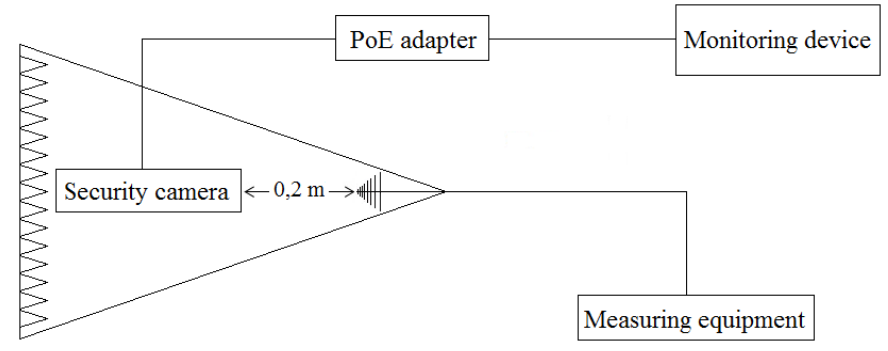

Figure 3. Disposition of measurement techniques and equipment under test.

\section{PROCESS OF MEASUREMENT}

As mentioned above, the measurement was carried out in different configurations of test devices, thus leading to new knowledge. To compare the results, the measurement was divided into two parts, the first was executed in the semianechoic chamber and the second was realized in GTEM. The 
tested parts remained the same for both tests. The frequency range of measuring equipment allowed to monitor the progress of interference in a broad spectrum. To measure, the quasipeak detector was used for more accurate results. The measurement procedure was identical for both tests and can be described by the following steps.

1) Preparing the Workplace: The first point involves the preparation of equipment used for testing.

2) Preparation and wiring of EuT: The test devices must be brought into operation and verified.

3) Execution of tests: The measurement must be performed in accordance with the standards.

4) Changing the configuration of EuT: This step necessitates the implementation of changes to the wiring of the test equipment.

5) Evaluation of assays: The last step consists of the elaboration and interpretation of the results.

\section{RESULTS OF MEASUREMENT}

\section{A. Semi-anechoic chamber}

The experiment was carried out according to the schemes shown in Figure 2. The antenna height was set to $2 \mathrm{~m}$, and a polarization was changed during the test. The green color always indicates the vertical position of the antenna; on the contrary, the violet color denotes the horizontal position.

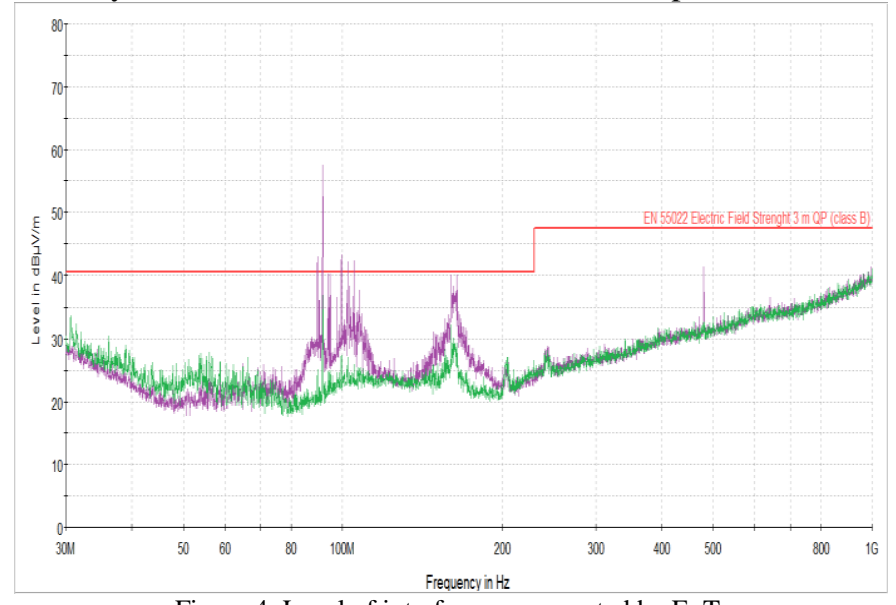

Figure 4. Level of interference generated by EuT.

As can be seen in Figure 4, the highest level of interference is recorded in the frequency range between 80 and $100 \mathrm{MHz}$ due to the parasitic interference of UTP. Interference corresponding to the IP camera and PoE adapter is in the range from 80 to $270 \mathrm{MHz}$. The maximum and minimum values obtained during the measurement are as follows:

1) Horizontal polarization of antenna

- maximum level: $56.112 \mathrm{~dB} \mu \mathrm{V} / \mathrm{m}(91.711 \mathrm{MHz})$,

- minimum level: $16.440 \mathrm{~dB} \mu \mathrm{V} / \mathrm{m}(47.936 \mathrm{MHz})$.

2) Vertical polarization of antenna

- maximum level: $37.238 \mathrm{~dB} \mu \mathrm{V} / \mathrm{m}(91.711 \mathrm{MHz})$,

- minimum level: $18.002 \mathrm{~dB} \mu \mathrm{V} / \mathrm{m}(77.991 \mathrm{MHz})$.

Location of PoE adapter outside the semi-anechoic chamber has the effect of reducing the level of electromagnetic interference except in areas of parasitic interference. This phenomenon is logical because the adapter processes the input voltage to a lower, thus resulting in a generation of disturbance.

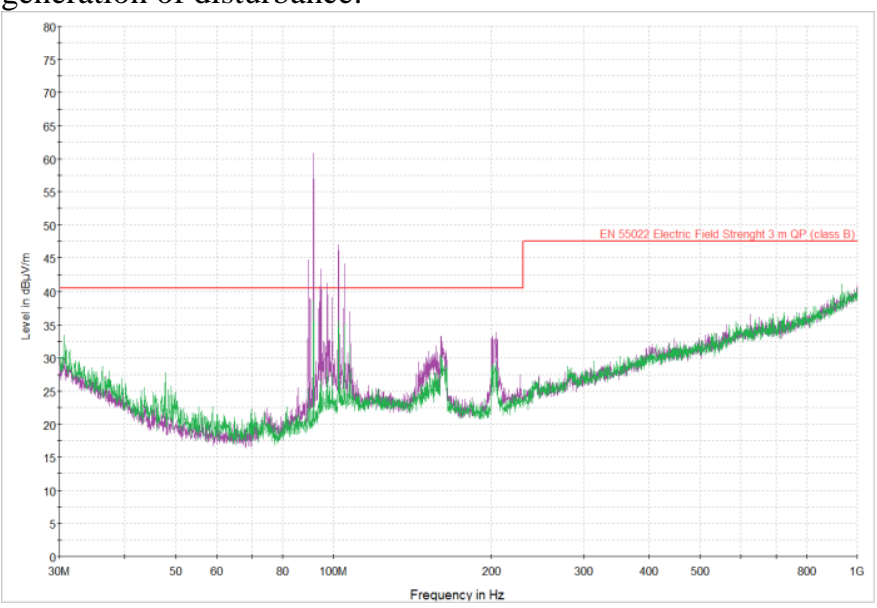

Figure 5. Level of interference generated only by IP camera

The maximum and minimum values of electromagnetic interference are following:

3) Horizontal polarization of antenna

- maximum level: $63.276 \mathrm{~dB} \mu \mathrm{V} / \mathrm{m}(91.711 \mathrm{MHz})$,

- minimum level: $16.291 \mathrm{~dB} \mu \mathrm{V} / \mathrm{m}(68.013 \mathrm{MHz})$.

4) Vertical polarization antenna

- maximum level: $42,515 \mathrm{~dB} \mu \mathrm{V} / \mathrm{m}(986.442 \mathrm{MHz})$,

- minimum level: $17.019 \mathrm{~dB} \mu \mathrm{V} / \mathrm{m}(77.168 \mathrm{MHz})$.

\section{B. GTEM cell}

The EuT and measuring equipment were connected according to the scheme presented in Figure 3. In contrast to the semi-anechoic chamber, GTEM cell does not have a movable antenna, therefore, the output of the test was specified by one course. RF attenuator was set to $10 \mathrm{~dB}$ and the value of the bandwidth indicated $120 \mathrm{kHz}$. The $\mathrm{x}$-axis always displays the frequency in $\mathrm{Hz}$, conversely, the $\mathrm{y}$-axis shows the values in $\mathrm{dB} \mu \mathrm{V} / \mathrm{m}$.

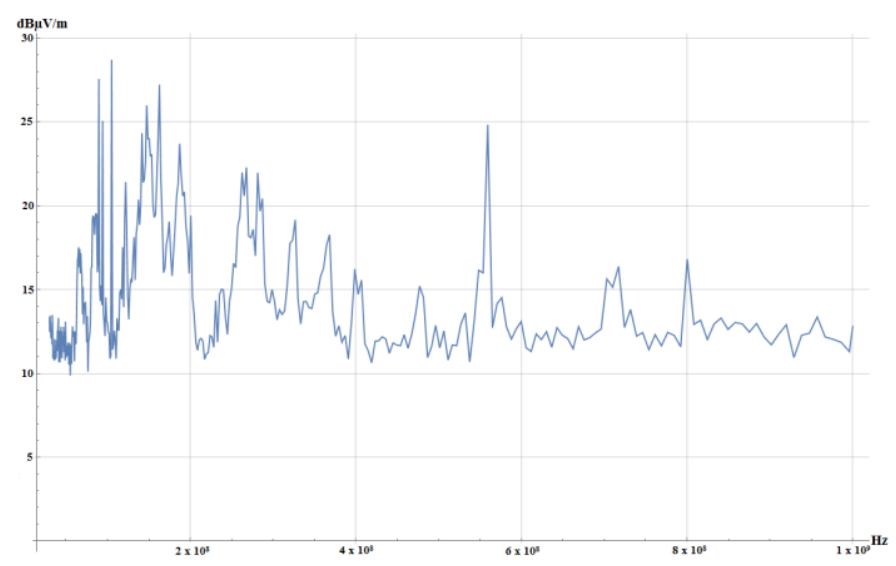

Figure 6. Level of interference generated by EuT in GTEM cell.

Figure 6 shows that the waveform of interference is similar to that obtained by testing in the semi-anechoic chamber. However, the set parameters of both test devices are varied which is reflected in the results. Level of electromagnetic interference reaches the following extreme values: 
- maximum level: $28.72 \mathrm{~dB} \mu \mathrm{V} / \mathrm{m}(105.103 \mathrm{MHz})$,

- minimum level: $9.87 \mathrm{~dB} \mu \mathrm{V} / \mathrm{m}(55.046 \mathrm{MHz})$.

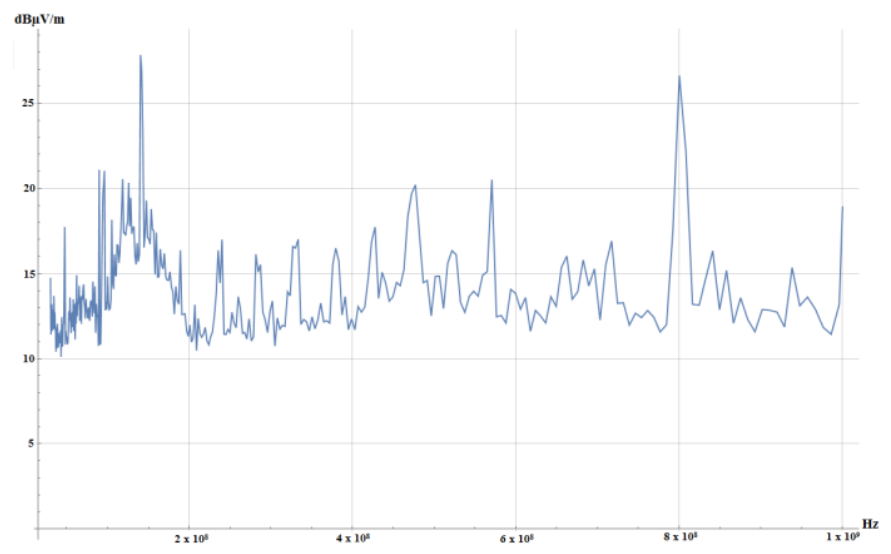

Figure 7. Waveform of electromagnetic interference without PoE adapter.

As can be seen in Figure 7, removing the PoE adapter from GTEM cell had a significant impact on the entire course of the interference; nevertheless, it is necessary to take into account the fact that the power adapter cable was not shielded. Maximum and minimum values of disturbance are as follows:

- maximum level: $27.85 \mathrm{~dB} \mu \mathrm{V} / \mathrm{m}(140.260 \mathrm{MHz})$,

- $\quad$ minimum level: $10.11 \mathrm{~dB} \mu \mathrm{V} / \mathrm{m}(42.923 \mathrm{MHz})$.

\section{CONCLUSION}

It should be noted that this study has been primarily concerned with the analysis and comparison of electromagnetic interference in the near and far field. The main problem of electronic and electrical devices related to electromagnetic compatibility are the transmission paths and power supplies. As the results of measurement show, unless these parts have quality shielding constitute the majority of disturbance. Currently, the greatest emphasis is placed on electromagnetic susceptibility; nonetheless, the interference generated by the information technology can provide key knowledge about the device. The analyzed device may be considered as safe because it meets the requirements specified by standards.

In addition, the paper presents a comparison of two different techniques to measure electromagnetic compatibility.
Although the full potential of both devices was not realized, each of them showed some advantages and efficiency for different situations. However, both devices can be interchangeable because their parameters are fully competitive. The tests were focused only on one type of security cameras or one type of safety applications, despite this fact, they give findings for further research in the field.

\section{ACKNOWLEDGMENT (HEADING 5)}

The work was funded with the support of the Internal Grant Agency of Tomas Bata University under the project No. IGA/CebiaTech/2016/005, and support of research project NPU I No. MSMT-7778/2014 by the Ministry of Education of the Czech Republic.

\section{REFERENCES}

[1] SVAČINA, Jiří. Electromagnetic compatibility: principles and notes. Issue No. 1. Brno: University of Technology, 2001, 156 p. ISBN 8021418737. (in Czech)

[2] Encyclopedia electromagnetic compatibility [online]. 2009 [cit. 201504-27]. Available at: http://www.radio.feec.vutbr.cz/emc/. (in Czech)

[3] KOVÁŘ, Stanislav, Jan VALOUCH, Hana URBANČOKOVÁ and Milan ADÁMEK. Electromagnetic interference of CCTV. In: The International Conference on Information and Digital Technologies 2015. Slovakia, Žilina, 2015. p. 161-166. ISBN 978-1-4673-7185-8. 6 p.

[4] OTT, Henry. Electromagnetic Compatibility. USA, Hoboken: WILEY,2009. ISBN978-0-470-18930-6. 844 p.

[5] KOVÁČ, D., KOVÁČOVÁ, I., KAŇUCH, J. EMC in terms of theory and application. Issue No. 1. Prag: BEN, 2006. ISBN 80-7300-202-7. 216 p. (in Czech)

[6] VALOUCH, Jan. Integrated Alarm Systems. In Computer Applications for Software Engineering, Disaster Recovery, and Business Continuity. Series: Communications in Computer and Information Science, Vol. 340, 2012, XVIII. Berlin: Springer Berlin Heidelberg, 2012. Chapter, p. 369 -379. ISSN 1865-0929.

[7] VALOUCH, Jan. Electromagnetic Compatibility of Alarm Systems Legislative and Technical Requirements. In :Security Magazin. Issue No 106, 2/2012. Praha: Security Media, 2012. ISSN 1210- 8273. p. 32-36.

[8] VALOUCH, Jan. Electromagnetic Compatibility of CCTV.Alarm Focus. Issue. No 2, 2/2013. Brandýs nad Labem: Orsec, 2013, p. 22- 23. ISSN 1805-9007. (in Czech)

[9] VALOUCH, Jan. Technical requirements for Electromagnetic Compatibility of Alarm Systems. In: International Journal of Circuits, Systems and Signal Processing. Volume 9. USA, Oregon: North Atlantic University Union, 2015. p. 186 - 191. ISSN: 1998-4464. p. 6. 